\title{
APROXIMACIÓN AL VALOR ECONÓMICO DE LOS HURTOS COMETIDOS EN LA CIUDAD DE CARTAGENA DURANTE EL PERIODO 1998-2007*1
}

\author{
Approximation to the economic value of thefts committed in the city of \\ Cartagena during the period 1998-2007
}

\section{Fabio Atencia Meza² Uriel Muñoz Guerrero² Fredi Goyeneche González ${ }^{3}$}

\section{RESUMEN}

Actualmente, los hurtos se han venido constituyendo en una de las principales preocupaciones para las autoridades y los habitantes de la ciudad de Cartagena, dado que las cifras de este tipo de actividad delictiva, se han venido incrementando de manera alarmante en los últimos años. De esta manera, el objetivo del presente artículo es realizar una estimación aproximada del valor económico de los hurtos cometidos dentro del área urbana de la ciudad. Para lo cual, el estudio toma en consideración el número de casos denunciados como hurtos, según las modalidades de hurto a residencias, a personas, a comercio, piratería y bancos y su respectivo valor monetario de las pérdidas materiales a causa de este fenómeno. Generalizando así con un análisis de la evolución y el comportamiento que ha tenido este tipo de actividad delictiva según la clase para el periodo de 1998-2007. Donde se encuentra que los hurtos a personas, a comercios y residencias concentran más del $90 \%$ de la totalidad de dinero hurtado en Cartagena y son las modalidades de hurtos que en su mayoría cobijan el valor total de las pérdidas materiales en este concepto. El valor económico de los hurtos ocurridos en la ciudad de Cartagena durante el periodo respectivo a precios del 2007 corresponde a 71.468 millones de pesos en total.

Palabras Claves: Valor Económico, Hurtos a Persona, Hurto a Residencia, Hurto a Comercio, Hurto a Bancos, Piratería, Cartagena de Indias.

Clasificación JEL: K24, N36, O18, Y10.

\footnotetext{
* Documento presentado en las Jornadas de Investigación de la Facultad de Ciencias Económicas de la Universidad de Cartagena.

${ }^{1}$ Este artículo fue realizado tomando como referencia la tesis de Hernando Padaui, para obtener el título de magíster en Desarrollo Social.

2 Programa de Economía de la Facultad de Ciencias Económicas- Universidad de Cartagena. Correo electrónico: fatencia574@hotmail.com

${ }^{3}$ Director del Centro de Observación y Seguimiento del Delito (COSED). Correo electrónico: fregoy@ hotmail.com
} 


\begin{abstract}
Currently, thefts have been becoming one of the main concerns for the authorities and the inhabitants of the city of Cartagena, given that the figures of this type of criminal activity, have been increasing alarmingly in recent years. In this way, the objective of this article is to make an approximate estimate of the economic value of thefts committed within the urban area of the city. For which, the study takes into account the number of cases reported as thefts, according to the modalities of theft to residences, to people, to commerce, piracy and banks and their respective monetary value of the material losses due to this phenomenon. Generalizing this way with an analysis of the evolution and the behavior that this type of criminal activity has had according to the class for the 1998-2007 period. Where it is found that the thefts to people, shops and residences concentrate more than $90 \%$ of the total amount of money stolen in Cartagena and are the types of thefts that mostly cover the total value of material losses in this concept. The economic value of the thefts that occurred in the city of Cartagena during the respective period at 2007 prices corresponds to 71,468 million pesos in total.
\end{abstract}

Keywords: Economic Value, Theft to Person, Theft to Residence, Theft to Commerce, Bank Theft, Piracy, Cartagena de Indias.

JEL Classification: K24, N36, O18, Y10.

\title{
Introducción
}

A largo de la historia, Cartagena de indias se ha convertido en ciudad inmemorable, llena de encantos y maravillas hasta el punto que ha sido catalogada según la UNESCO en 1984, patrimonio histórico y cultural de la humanidad. Posee a nivel nacional e internacional la imagen de una ciudad tranquila y armoniosa, lo cual se convierte en un atractivo para el turismo desde todos los ángulos. Esta sería la cara positiva que toda ciudad a nivel mundial poseería, sin antes saber que detrás de toda esta gracia se presentan situaciones funestas y tristes que dañan su fachada floreciente de ciudad amable y próspera. Se presenta ahora la Cartagena llena de grandes problemas económicos y sociales, sumados a los altos índices de inseguridad de su comunidad que va desde elevadas tasas de homicidios hasta la profundización de los indicadores de hurtos o atracos en diferentes modalidades. Para este caso, la siguiente investigación hace un gran recorrido a la problemática que ostenta la ciudad en cuanto a las pérdidas materiales por causa del fenómeno de los hurtos. Es claro, que los fenómenos de inseguridad en la ciudad generan efectos negativos para el desarrollo integral de la misma, por lo tanto, se hace 
necesario que se tomen medidas inmediatas para frenar este tipo de situaciones, para lo cual se requiere la intervención rápida y oportuna de los entes encargados de mantener la seguridad y la tranquilidad de la comunidad, sin dejar de lado que esta problemática está asociada a una variedad de factores que también hay que enfrentar.

En las últimas décadas, Cartagena de indias se ha caracterizado por mantener diversidad de actividades económicas en términos productivos, escalafonado en sus tres principales sectores económicos: industria, comercio y turismo, aspectos que de una u otra forma, podrían incidir de manera importante en el incremento de actividades delictivas como las tratadas en esta investigación, las cuales acompañan a otras como el vandalismo, este último asociado a otros fenómenos como la drogadicción y las pandillas. De esta manera se puede apreciar el panorama grotesco que presenta la ciudad en términos de inseguridad, que de igual forma, pueda que estos aspectos no solo afecten en lo social, sino también de manera directa e indirecta a la economía local. Desde esta perspectiva se intenta darles una valoración económica a los hurtos cometidos en la ciudad durante el periodo de 1998-2007. A partir de la estimación del valor de los hurtos cometidos a residencia, personas, bancos, comercio y piratería, determinando así el total en cifras exactas de las pérdidas materiales causadas por este tipo de fenómeno.

Para tal caso, el estudio iniciara con una breve introducción de la situación actual de Cartagena de indias en términos de inseguridad para efectos de las pérdidas materiales por hurtos, en segundo lugar una magnifica revisión literaria muy asociada al contexto que se estudia, mientras que en aspecto tenemos la metodología que permitirá mostrar la tipología del estudio a seguir, desembocando así en cuarta medida en el componente de los resultados, parte de mayor importancia en la investigación y finalmente culmina con la muestra de la base bibliográfica utilizada durante la elaboración del trabajo.

\section{Revisión de la literatura}

Para explicar valor económico de los hurtos cometidos en la ciudad de Cartagena durante el periodo 1998-2007, se tuvo en cuenta una gran variedad de investigaciones de carácter nacional e Internacional, las cuales sirvieron de apoyo y guía en la realización del actual trabajo y, además, permitieron consolidar algunos procedimientos de trabajo para abordar la temática actual. 
En este ámbito, es destacable la revisión literaria realizada por Hernando Padaui, (2009), en su trabajo "Valoración económica del delito y su impacto en el desarrollo local de Cartagena, 1998 2007" en la cuya investigación se encontró que en el año 1998 dado la cantidad de homicidios cometidos, la sociedad perdió un total de 8.107 años de vida productiva y de aportación a la riqueza y la producción colectiva. Esta cifra se mantiene un crecimiento del 3\% anual hasta el 2007, hasta llegar a 9.029 años de vida productiva perdidos en este último año, además de que la valoración económica de los homicidios utilizando el método del PIB per cápita, arroja valores mucho más altos que para el caso del Salario Nominal. Se puede observar, por ejemplo, que estos costos han oscilado entre los \$49.643 millones en 1998 hasta alcanzar valores de \$67.910 millones en el 2007. Para este investigación es importante resaltar la desagregación de trabajo a nivel internacional como Brand y Price (2000), los cuales elaboraron una estimación de los delitos en Inglaterra y gales para el periodo de 1999 - 2000, haciendo su análisis con base en las inversiones en seguridad, pérdidas materiales, daños físicos y emocionales, gastos médicos e inversión en el sistema de justicia; los resultados arrojados muestran que para ese periodo la estimación de los costos totales del delito fue de 60 mil millones de libras esterlinas.

En este orden también se destacar para Chile, el trabajo realizado por Iván Silva Lira, el cual preparó un documento titulado “Costos Económicos De Los Delitos, Niveles De Vigilancia Y Políticas De Seguridad Ciudadana En Las Comunas Del Gran Santiago”. Empieza preguntándose cuál es el monto de los ahorros que se podrían generar en términos de pérdidas materiales, perdidas de vida, atención a heridos y tiempo de trabajo etc. versus el gasto de inversión que habría que hacer para que los mencionados ahorros se produjeran. La respuesta la halla afirmando que se trata de un análisis de costo beneficio que puede resultar en inversiones con una alta tasa de rentabilidad social.

Tenemos el caso del trabajo de Catalina Mertz denominado "Costo De La Delincuencia: Chile 1996" hace un análisis de costos de prevención, aplicación de sanciones y el costo alternativo de los recursos del Estado. En dicho estudio se calculó que éste llegaba en 1994 a US\$1.290 millones. Dos años más tarde, haciendo la estimación de 1996, se mostró que éste había aumentado a US\$ 1.480 millones. La investigación se desarrolla contabilizando los costos directos e indirectos a consecuencia del crimen, valorando económicamente las perdidas según el agente al que perjudican. De esta manera son identificados los costos de las víctimas, los delincuentes y la sociedad. 
Dentro de la literatura revisada para el caso de Colombia, encontramos también, investigaciones realizadas por el Departamento Nacional de Planeación (DNP) entre ellas, "Los Costos Económicos De La Criminalidad Y La Violencia En Colombia: 1991-1996"; elaborado por Edgar Trujillo Ciro y Martha Elena Badel Rueda. En este trabajo se plantea que la acción criminal desencadena costos económicos directos que son representados por las pérdidas de capital físico y humano; además de costos indirectos generados por la incertidumbre, que se traducen en desestimulo o desvío de la inversión, asignación ineficiente de recursos, y aumento de los costos de transacción. Por lo tanto, la criminalidad disminuye la productividad de la economía además de frenar su crecimiento. Realiza un análisis descriptivo de la criminalidad y su evolución en Colombia desde los años sesenta, mostrando que los delitos de mayor participación son los delitos contra el patrimonio económico y contra la vida. Luego continúa con la caracterización de las víctimas de homicidio en el país, señalando que para 1996 ocurrió un total de 26,510 homicidios lo que equivale a una tasa de homicidios de 67 por cada 100 mil habitantes. Por sexo, el 93\% de las víctimas fueron hombres y el restante 7\% mujeres. Posteriormente, describe de manera breve los costos de la criminalidad, diferenciando entre costo bruto y costo neto; el primero hace alusión al que asume la víctima del delito, por ejemplo, el valor de un objeto robado o destruido o el pago de un rescate; mientras que el costo neto se refiere al costo macroeconómico, un acto delictivo tiene costos macroeconómicos netos si disminuye el valor agregado actual o futuro de una economía.

Destacamos también a Mauricio Rubio, con su trabajo "Los Costos De La Violencia En Colombia”, donde señala que, en las últimas décadas, a nivel internacional, ha surgido interés de la disciplina económica por el crimen y la acción estatal para enfrentarlo. Rubio pretendió enriquecer el debate sobre prioridades de acción pública en materia de prevención y control de la violencia. Además, discute un impacto hasta el momento ignorado de la violencia colombiana y es el de su efecto regresivo sobre la distribución del ingreso. Por último, se aborda el tema del efecto de la violencia sobre la eficiencia económica y concluye que la violencia está poniendo en peligro la viabilidad de la economía colombiana y que junto con la falta de acciones públicas realistas y efectivas, han generado una progresiva privatización de bienes públicos por excelencia, como la seguridad y la justicia, y sumado a esto; los costos de transacción y las oportunidades perdidas se da un fuerte impacto sobre el capital físico, humano y social. 
En este campo también es importantísimo mencionar a Goyeneche Fredi, con su trabajo titulado “Aproximación A Determinantes De La Violencia Homicida En Cartagena” en el cual se realiza una caracterización de los casos de homicidios ocurridos hasta el año 2007, y relaciona su causalidad e incidencia con aspectos sociales de relevancia que hacen entender al final que existe una relación territorial entre la incidencia de delitos y las zonas de mayor marginalidad de la ciudad. Afirma que "la delincuencia organizada, dentro de la cual se ubican los homicidios por hurto, por sicarito y ajuste de cuentas, resulta ser la de mayor frecuencia en todo el 2006 reportando 146 de los casos de homicidios, cifra superior en 25 casos a los reportados durante el 2005.

De igual manera, tenemos el trabajo desarrollado por Jerry Pardo y Oswaldo Mármol, titulado “Costos económicos de los homicidios en Cartagena y su impacto en la inversión. 1995 - 2005”, el cual estima los costos económicos causados por la violencia homicida para el periodo referenciado y además estima un impacto que dichos costos tendrían sobre los niveles de inversión directa en el Distrito. Como conclusión relevante encuentra que el Valor total de toda la serie de costos económicos a precios del 2005 alcanzó una cifra igual a \$530.778 millones de pesos y que la violencia homicida, para el caso de Cartagena, ejerce una influencia inversa sobre los niveles de inversión en capital físico, aunque encuentran de igual forma que dicha influencia, para el periodo estudiado, resulta no ser altamente significativa.

Sobresale de igual forma el trabajo desarrollado por Luz Adriana Doria Arteaga y Milena Isabel Mejía Martínez llamado “Costos Económicos De La Violencia Intrafamiliar En La Ciudad De Cartagena.”. Donde se encuentra que la violencia con su multiplicidad de hechos, manifestaciones, actores, victimas y escenarios, constituye hoy en día una de las realidades más preocupantes de la vida nacional. En el primer capítulo se describe de manera general la situación social y económica de la ciudad de Cartagena, después se describe la situación de la violencia intrafamiliar de la ciudad donde se resalta la influencia de las características socioeconómicas de los hogares cartageneros en la en la periodicidad de la violencia intrafamiliar para el periodo 2005. El trabajo se basó en encuestas de victimización y se encontró en el que "el costo total del tratamiento por hogar, según la naturaleza de la violencia intrafamiliar es: violencia verbal $\$ 16.940 .000$, violencia física y verbal simultáneamente $\$ 13.416 .000$ y la sicológica $\$ 49.735 .000$, violencia física $\$ 9.460 .000 ”$. 


\section{Metodología}

La metodología implementada en la presente investigación es de carácter investigativo-descriptivo donde se busca hacer una aproximación al valor económico de los hurtos cometidos en la ciudad de Cartagena durante el periodo 1998-2007, para lo cual se establecen indicadores que permiten determinar la cuantía para esta problemática, que afecta el desarrollo de la sociedad Cartagenera. En este sentido, se tomó en consideración como población objeto de estudio la ciudad de Cartagena en todo el ámbito social, donde se realiza una estimación aproximada del valor económico de los hurtos cometidos en la ciudad, donde las cifras son obtenidas a partir de las denuncias realizadas por los afectados por este tipo de actividad delictiva, dadas las diferentes modalidades de hurto y robos. El diseño del estudio es de carácter no experimental, puesto que no se construyó una situación, sino que se estudiaron situaciones ya existentes.

La información de la presente investigación fue obtenida a partir de una variada fuente bibliográfica (fuente secundaria) e informes de la Policía Nacional seccional Cartagena, para la cual se realizaron cálculos estadísticos mediante el software Microsoft Office Excel 2007, donde se obtuvieron cuadros y graficas que permitieron establecer el análisis del valor económico aproximado de los hurtos cometidos en la ciudad de Cartagena durante el periodo de estudio.

\section{Resultados}

Para hacer este análisis, se toma como fuente de información las denuncias hechas sobre los hurtos cometidos que reposan en el Centro de Investigaciones Criminalísticas (CIC) de la Policía Nacional de Bolívar, mediante las cuales se pudo sustraer la cuantía las victimas reportan haber perdido en el momento del hurto.

Las autoridades competentes asignan el valor a los bienes o activos perdidos de acuerdo con el testimonio dado por la víctima y de la modalidad de hurto, es decir, la víctima es quien reporta la cantidad de bienes usurpados y el monto en que cada uno de estos están valorado para luego hacer un consolidado que permita saber con exactitud el valor monetario perdido. Para entender de una mejor manera esta situación se toma como ejemplo una persona, la cual en el momento de un hurto pierde dinero y algunos accesorios, en este caso el valor monetario total de la pedida es la suma del dinero y el valor monetario de los bienes perdidos, lo mismo ocurre con los hurtos a residencia y al sector comercial. 
En el caso de entidades del sector financiero es normal que la perdida sea monetaria, por lo cual al momento de hacerse el estimativo monetario de este hurto la cantidad será exactamente la cantidad hurtada. Finalmente, los casos de piratería terrestre, se cuantifican de acuerdo con el valor económico de los bienes incautados o decomisados. Es de vital importancia aclarar que faltan algunos datos que se requieren para armar esta investigación porque no se encuentran disponibles en la fuente que suministra los datos, característica que se evidenciará en el transcurso del estudio. Por tal razón en adelante notara el lector que para describir el comportamiento de las cifras en el tiempo no sean mencionados estos años, pero los resultados finales si están enmarcados en el periodo propuesto desde el inicio a la investigación ya que esta información es solo un componente de los resultados mas no el resultado mismo. Hechas estas salvedades, seguimos con el análisis de los resultados de esta investigación.

En la tabla No. 1 se muestra el número de casos denunciados como hurtos según sus modalidades.

Tabla1. Casos de hurtos según tipo. Cartagena 2000 - 2007

\begin{tabular}{|c|c|c|c|c|c|c|c|c|c|c|}
\hline AÑ & $\mathbf{1 9 9 8}$ & $\mathbf{1 9 9 9}$ & $\mathbf{2 0 0 0}$ & $\mathbf{2 0 0 1}$ & $\mathbf{2 0 0 2}$ & $\mathbf{2 0 0 3}$ & $\mathbf{2 0 0 4}$ & $\mathbf{2 0 0 5}$ & $\mathbf{2 0 0 6}$ & $\mathbf{2 0 0 7}$ \\
\hline Hurto Personas & ND & ND & 902 & 951 & 1289 & 1092 & 969 & 1027 & 1956 & 1417 \\
\hline Hurto Residencias & ND & ND & 451 & 400 & 312 & 353 & 400 & 332 & 576 & 329 \\
\hline Hurto Comercio & ND & ND & 587 & 681 & 526 & 493 & 423 & 388 & 699 & 385 \\
\hline Piratería & ND & ND & 6 & 15 & 4 & 10 & 7 & 7 & 5 & 6 \\
\hline Bancos & ND & ND & 0 & 2 & 7 & 13 & 2 & 0 & 0 & 0 \\
\hline
\end{tabular}

Fuente: Policía Nacional

Teniendo en cuenta esto podemos notar que la modalidad de hurto más concurrida durante el periodo de estudio es el "hurto a personas", en el cual se presentaron 9.603 casos, es decir, 9.603 personas denunciaron ser víctimas de hurtos en Cartagena, para un promedio de 1.200 casos por año siendo 2002, 2006 y 2007 los años en que más se presentaron estos casos y claramente están por encima del promedio para Cartagena.

En una segunda posición a parecen los "hurtos al comercio" con 4.182 casos reportados, luego se muestran los "hurtos a residencias" con 3.153 reportes, mientras que los casos por piratería y hurtos a banco no superan los 20 casos por año y juntos no suman más de 70 casos en periodicidad total. Para estas dos últimas modalidades de hurto, el número de casos presentados es bajo y en algunos años nulos, como se puede notar en la modalidad "hurtos a banco" en la cual no se presentan casos para los 
años 2005, 2006 y 2007, debido a lo riesgoso que es llevarlos a cabo por la extrema vigilancia prestada por las autoridades tanto públicas como privadas.

Los hurtos a personas suelen ser los más frecuentes, como se observa en la Tabla No. 1 en los últimos ocho años 9.603 personas han denunciado ser víctimas de un hurto en Cartagena, para un promedio de 1200 casos denunciados por año, siendo los años más representativos 2006, 2007 y 2002 , claramente por encima del promedio. Por su parte Las denuncias por hurtos a comercio en los últimos ocho años sumaron 4182 casos, siendo inferior en más de 5421 casos a los registrados por hurto a personas, igual relación mantienen los hurtos a residencia, que durante todo este periodo totalizan 3153 denuncias, mientras que por piratería y hurtos a bancos las denuncias no superan los 20 casos por año y no suman más de 70 casos en la periodicidad total. Para estas dos modalidades de hurtos, el número de casos y denuncias presentadas son bajas e inclusive nulas, como efectivamente se puede observar en los hurtos a bancos de los años 2005, 2006 y 2007, entre otras razones por el alto riesgo implícito a la realización de esta actividad delictiva, debido a la alta vigilancia que ejercen las autoridades tanto públicas como privadas.

Al examinar la cuantía o valor monetario de las perdidas por hurtos encontramos que las cantidades más significativas se presentaron en la modalidad de "hurto a comercio" con un total de $\$$ 20.686`000.000, valor que representa el 35.3\% del valor monetario total de todos los hurtos por todas las categorías sin ser esta la que presentó el mayor número de casos reportados. Como se puede notar luego de este análisis, la mayor cuantía está determinada por el valor monetario de los bienes hurtados a los establecimientos comerciales y no al número de casos presentados, dicho de otra forma, hay más perdida de dinero en 4.182 casos de hurto a comercio que en 9.603 casos de hurto a personas, pues obviamente los establecimientos comerciales tienen más objetos de valor o dinero que hurtar que una persona.

Tabla 2. Valor monetario de las pérdidas materiales a causa de los hurtos. Cifras en Pesos. 2000 - 2007

\begin{tabular}{|c|c|c|c|c|c|c|}
\hline AÑOS & $\begin{array}{c}\text { Hurtos a } \\
\text { Personas }\end{array}$ & $\begin{array}{c}\text { Hurtos a } \\
\text { Comercios }\end{array}$ & $\begin{array}{c}\text { Hurtos a } \\
\text { Residencias }\end{array}$ & $\begin{array}{c}\text { Piratería } \\
\text { Terrestre }\end{array}$ & $\begin{array}{c}\text { Hurto a } \\
\text { Bancos }\end{array}$ & Total \\
\hline 1998 & ND & ND & ND & ND & ND & ND \\
\hline 1999 & ND & ND & ND & 0 & ND & -ND \\
\hline 2000 & 1.667 .256 .537 & 2.586 .093 .684 & 2.155 .861 .130 & 446.098 .511 & -ND & 6.855 .309 .861 \\
\hline
\end{tabular}




\begin{tabular}{|c|c|c|c|c|c|c|}
\hline 2001 & 1.757 .828 .123 & 4.252 .528 .701 & 1.912 .071 .956 & 1.115 .246 .276 & 45.722 .585 & 9.083 .397 .641 \\
\hline 2002 & 2.382 .587 .224 & 3.326 .911 .535 & 1.491 .416 .125 & 297.399 .007 & 160.029 .048 & 7.658 .342 .939 \\
\hline 2003 & 1.593 .870 .359 & 2.401 .294 .369 & 1.268 .594 .628 & 473.427 .450 & 153.518 .859 & 5.890 .705 .665 \\
\hline 2004 & 1.554 .456 .067 & 1.829 .528 .203 & 2.021 .782 .715 & 285.314 .521 & 189.400 .529 & 5.880 .482 .035 \\
\hline 2005 & 2.279 .178 .627 & 2.225 .897 .872 & 2.370 .828 .440 & 1.161 .400 .830 & 0 & 8.037 .305 .769 \\
\hline 2006 & 3.895 .823 .076 & 2.367 .718 .481 & 2.278 .673 .013 & 236.000 .000 & 0 & 8.778 .214 .570 \\
\hline 2007 & 2.619 .182 .387 & 1.696 .160 .253 & 1.572 .679 .184 & 446.098 .511 & 0 & 6.334 .120 .334 \\
\hline TOTAL & $\mathbf{1 7 . 7 5 0 . 1 8 2 . 3 9 9}$ & $\mathbf{2 0 . 6 8 6 . 1 3 3 . 0 9 8}$ & $\mathbf{1 5 . 0 7 1 . 9 0 7 . 1 9 1}$ & $\mathbf{4 . 4 6 0 . 9 8 5 . 1 0 6}$ & $\mathbf{5 4 8 . 6 7 1 . 0 2 1}$ & $\mathbf{5 8 . 5 1 7 . 8 7 8 . 8 1 4}$ \\
\hline
\end{tabular}

Fuente: Policía Nacional

En lo que respecta al hurto a residencia, también presenta una significativa cuantía la cual está valorada en $\$ 15.071^{\prime} 907.191$ correspondientes a 3.153 casos denunciados, es decir, el $25.7 \%$ del total del valor monetario de los hurtos para el periodo comprendido entre 2000 y 2007. En general los hurtos a personas, a comercios y residencias concentran más del $90 \%$ de la totalidad de dinero hurtado en Cartagena y son las modalidades de hurtos que en su mayoría cobijan el valor total de las pérdidas materiales en este concepto. La piratería y el hurto a bancos no son muy representativos en cuanto a número de casos denunciados y tampoco en cuanto al valor económico reportad.

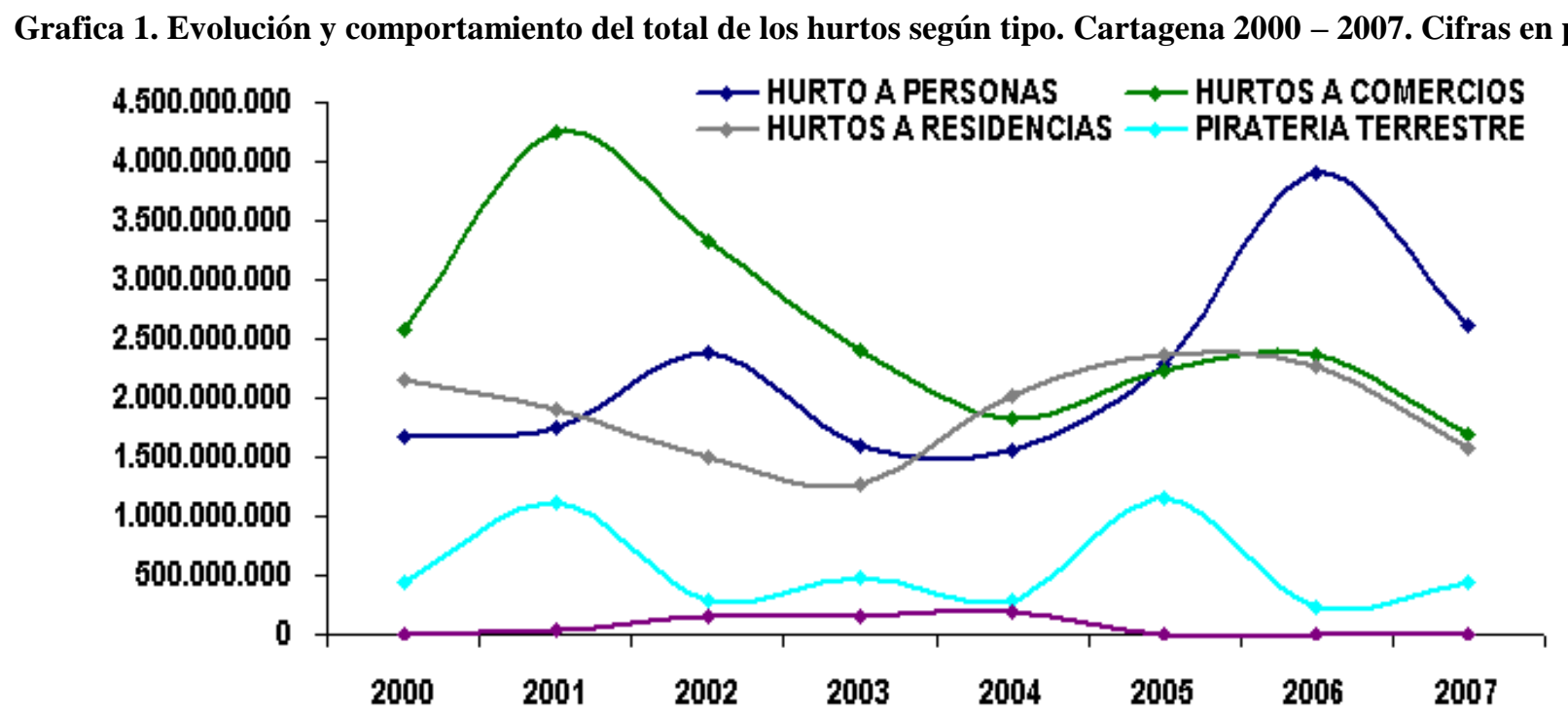

Fuente: Policía Nacional

Mediante la gráfica No. 1 podemos ver cómo ha sido el comportamiento y dinámica de las distintas modalidades de hurtos según el valor monetario perdido. Los hurtos a comercio son los más representativos, permaneciendo la mayor parte del tiempo por encima de los hurtos a residencias, a 
entidades financieras y la piratería. Pero en cuanto a los hurtos a personas solo son superiores hasta el año 2004, alcanzan una intercepción en el año 2005 para luego ser superados ampliamente en los dos años restantes (2006 y 2007). De manera general el valor económico de los hurtos a comercio registra una tendencia decreciente, registrando su mayor valor en el año de 2001 ubicándose en 4.252 millones de pesos, mientras que la cifra más baja la registra en el 2007, siendo de 1.696 millones de pesos.

En tanto que el valor económico de los hurtos a personas presenta una tendencia decreciente hasta el año 2004 para luego crecer hasta el 2006 alcanzando su mayor valor, 2.619 millones de pesos y disminuir en el 2007, pero aun siendo superior su cuantía económica a la de las otras 4 modalidades de hurtos.

El valor económico que registran los hurtos a residencia muestra una tendencia decreciente hasta el año 2003 pasando de 2.155 millones de pesos en el año 2000 a 1.268 millones en el 2003, luego inicia un comportamiento creciente hasta el año 2005 alcanzando su máximo valor, 2.370 millones de pesos, luego desciende has llegar a 1.572 millones de pesos en año 2007.

La modalidad de piratería terrestre que dentro de los delitos contra el patrimonio es considerado como un hurto. De acuerdo con la Grafica No. 1 su tendencia es oscilante con picos altos en los años 2001 y 2005 y con cuantías económicas relativamente bajas si las comparamos con las mostradas por las otras modalidades.

Pese a que ocurren aproximadamente 3 casos en promedio al año la modalidad de hurtos a bancos registra una importante cuantía, 548 millones de pesos en total en todo el periodo de estudio, valor que obviamente no es nada comparado con las cuantías económicas registradas en las otras modalidades de hurtos.

Lo que pretende este estudio es mostrar el valor económico que representa para las víctimas y para toda la sociedad la perdida que se sufre con los hurtos ya que para la víctima el hurto representa naturalmente una pérdida económica directa e inmediata, es por ello que a continuación se muestra el valor económico de estas pérdidas a precios corrientes de cada año y aprecios del 2007 con el fin de tener una idea de cuánto es el total que a la sociedad cartagenera le es arrebatada en montos del último año de investigación. 
Tabla 3. Valor total y anual de las pérdidas materiales a causa de los hurtos. Cartagena de Indias. 2000-2007. Cifras

\begin{tabular}{|l|l|l|}
\hline \multicolumn{2}{|c|}{ en pesos } \\
\hline AÑOS & \multicolumn{1}{|c|}{ Total } & \multicolumn{1}{|c|}{$\begin{array}{c}\text { Serie a Precios del } \\
\mathbf{2 0 0 7}\end{array}$} \\
\hline 1998 & ND & ND \\
\hline 1999 & ND & ND \\
\hline 2000 & 6.855 .309 .861 & 10.375 .196 .392 \\
\hline 2001 & 9.083 .397 .641 & 12.641 .199 .852 \\
\hline 2002 & 7.658 .342 .939 & 9.961 .657 .215 \\
\hline 2003 & 5.890 .705 .665 & 7.195 .405 .596 \\
\hline 2004 & 5.880 .482 .035 & 6.808 .452 .692 \\
\hline 2005 & 8.037 .305 .769 & 8.875 .187 .823 \\
\hline 2006 & 8.778 .214 .570 & 9.277 .694 .979 \\
\hline 2007 & 6.334 .120 .334 & 6.334 .120 .334 \\
\hline TOTAL & $\mathbf{5 8 . 5 1 7 . 8 7 8 . 8 1 4}$ & $\mathbf{7 1 . 4 6 8 . 9 1 4 . 8 8 2}$ \\
\hline
\end{tabular}

Fuente: Cálculos del autor con base en la policía Nacional

Como se puede observar si se trae el valor total de los hurtos para todo el periodo de estudio a precios del 2007 la cantidad económica incrementa pasando de 58.517 millones de pesos a 71.468 millones de pesos.

Grafica 2. Comparación de la serie de pérdidas materiales a causa de los hurtos a precios corrientes y a precios del 2007. Cartagena de Indias. 2000 - 2007. Cifras en pesos.

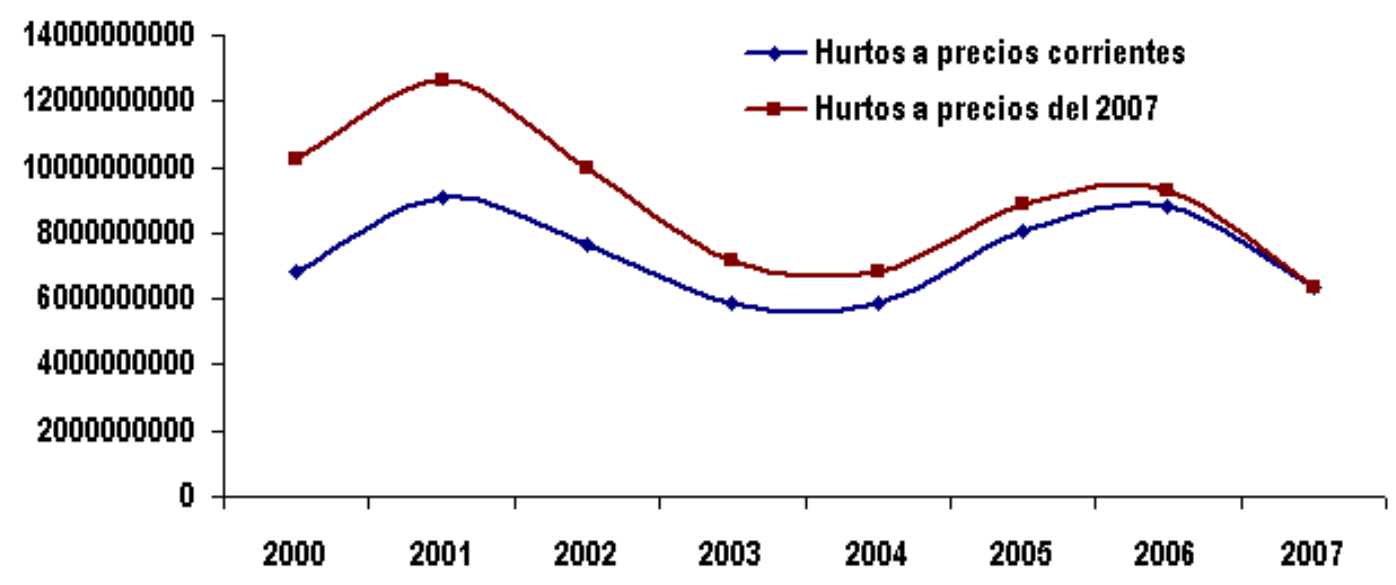

Fuente: Cálculos del autor con base en la policía Nacional 
Según la gráfica No. 2 que ilustra de mejor manera el comportamiento en el tiempo que registra el cálculo del valor económico de los hurtos en Cartagena a precios corrientes para cada año y a precios del 2007, en el año 2001 se registra el valor económico de los hurtos más alto de toda la serie seguido por el valor registrado en el 2006, pero en 2004 alcanza su valor más bajo, lo que hace que la tendencia se parta en dos: una tendencia creciente hasta el 2004 para luego incrementarse hasta llegar a los 9.277 millones de peso en el 2006 y finalmente descender en el 2007. De manera general, ambas cantidades mantuvieron el mismo ritmo y la misma tendencia a lo largo de todo el periodo de estudio.

\section{CONCLUSIONES}

- Los casos de mayor incidencia dentro del fenómeno de los hurtos para la ciudad de Cartagena es el hurto cometido a personas, el cual tuvo 9.603 casos reportados que representa aproximadamente 17.000 millones de pesos, pero aunque esta es la modalidad más concurrida es el hurto a comercio el que representa una mayor cuantía económica o valor monetario perdido con una cifra aproximada de 20.000 millones de pesos representados en 4.182 casos, es decir, que hay más perdida en menos casos reportados de hurtos a comercio que en el mayor número de reportes de hurtos a personas.

- 9.603 personas reportaron ser víctimas de hurtos en Cartagena, para un promedio de 1.200 casos por año siendo 2002, 2006 y 2007 los años en que más se presentaron estos casos y claramente están por encima del promedio para Cartagena.

- En cuanto a los hurtos a residencias, a bancos y piratería, el primero es quien presenta una cifra bastante significativa, en todo el periodo de estudio se perdieron aproximadamente 15.000 millones de pesos en esta modalidad de hurto mientras que los hurtos a entidades financieras son las menos significativas del estudio en cuanto a casos reportados y a valor monetario perdido con un valor aproximado de cerca 548 millones de pesos y la piratería que representa una modalidad de hurto con aproximadamente cerca de 4.000 millones de pesos en pérdidas.

\section{REFERENCIAS}

Banco de la Republica. "Informe de coyuntura económica regional departamento de Bolívar, ICER 2007" 
Blandón, Marlene. "Relación existente entre la pobreza y la violencia en Cartagena de indias. Periodo 2000-2006".

Cámara de Comercio de Cartagena. (1999). Cuadernos de coyuntura económica Cartagena de Indias, indicadores económicos de Cartagena. Tercer Trimestre de 1999 No 3, noviembre de 1999.

Centro de Investigaciones Criminalísticas (CIC) de La Policía Nacional del Departamento de Bolívar.

COSED. (2007). Informe Anual de MCT.

Goyeneche, Fredi. (2007). “Aproximación interpretativa a determinantes de la violencia en Cartagena”.

Pardo Jerry y Mármol Oswaldo. (2007). “Costos económicos de los homicidios en Cartagena y su impacto en la inversión. 1995-2005.

Rubio, Mauricio. Los costos de la violencia en Colombia. Centro de estudios sobre desarrollo económico. Documento de trabajo N. ${ }^{\circ} 11$

Silva Lira, Iván (2000). Costos económicos de los delitos, niveles de vigilancia y políticas de seguridad ciudadana en las comunas del Gran Santiago, Santiago de Chile Enero del 2000. P. 47

Policía Nacional - DIJIN y Reportes de evaluación del DNP.

Rubio, Mauricio. (2000). "Los Costos de la violencia en América Latina”.

Trujillo Ciro, Edgar. Badel Rueda, Martha Elena. (1998). Los costos económicos de la criminalidad y la violencia en Colombia: 1991-1996. Archivos de macroeconomía. Documento 76, 10 de marzo de 1998 